\title{
INITIAL PERFORMANCE OF THE BABAR EXPERIMENT
}

\author{
OWEN R. LONG (FOR THE BABAR COLLABORATION) \\ University of California, Santa Barbara, CA 93105, USA \\ E-mail: owen@slac.stanford.edu
}

\begin{abstract}
We present the initial performance of the BaBar Experiment, as of April 2000. The PEP-II machine has reached a peak instantaneous luminosity of $1.95 \times$ $10^{33} \mathrm{~cm}^{-2} \mathrm{~s}^{-1}$ and delivered an integrated luminosity of $7 \mathrm{fb}^{-1}$ in the first 11 months of operation, of which $6.5 \mathrm{fb}^{-1}$ has been logged by the BaBar experiment. This large data set has allowed us to study the performance of our detector in great detail and develop our physics analysis tools. The initial performance of each major detector subsystem is reviewed. We conclude with some preliminary analysis results and projections.
\end{abstract}

For an overview of the physics motivation and experimental challenges involved in measuring $C P$ violation in the $B$ system, we refer the reader to the lectures presented by Pat Burchat in these proceedings. We will assume that the reader of this report has a basic understanding of experimental high energy physics.

\section{The PEP-II asymmetric $B$ factory}

The PEP-II asymmetric $B$ factory resides at the Stanford Linear Accelerator Center (SLAC) in Menlo Park, California. The high design luminosity of $3 \times 10^{33} \mathrm{~cm}^{-2} \mathrm{~s}^{-1}$ is achieved through high currents and strong focusing. A Snowmass year $\left(10^{7} \mathrm{~s}\right)$ of running at the design luminosity will yield 30 million $B$ meson pairs, which meets the high statistics requirement for $C P$ asymmetry measurements. The center-of-mass system is boosted in the lab $(\beta \gamma=0.56)$ through unequal beam energies in order to have an observable difference in the flight length of the two $B$ hadrons $(\langle\delta z\rangle \approx 250 \mu \mathrm{m})$, and thus an observable $\delta t$.

Commissioning of the high energy ring (HER) of the PEP-II machine began in May of ' 97 . In March of ' 99 , commissioning was put on hold to install the BaBar detector. By this point, PEP-II had already achieved a luminosity of $5.2 \times 10^{32} \mathrm{~cm}^{-2} \mathrm{~s}^{-1}$ with 786 bunches. First collisions were recorded with the BaBar detector in May of ' 99 , and PEP-II achieved a then world record luminosity of $1.4 \times 10^{33} \mathrm{~cm}^{-2} \mathrm{~s}^{-1}$ in November of ' 99 . The current PEP-II record is $1.95 \times 10^{33} \mathrm{~cm}^{-2} \mathrm{~s}^{-1}$. Figure 1 shows the integrated and daily luminosity history of PEP-II and the BaBar experiment. The inactive period near October ' 99 was a planned shutdown to install the remainder of 

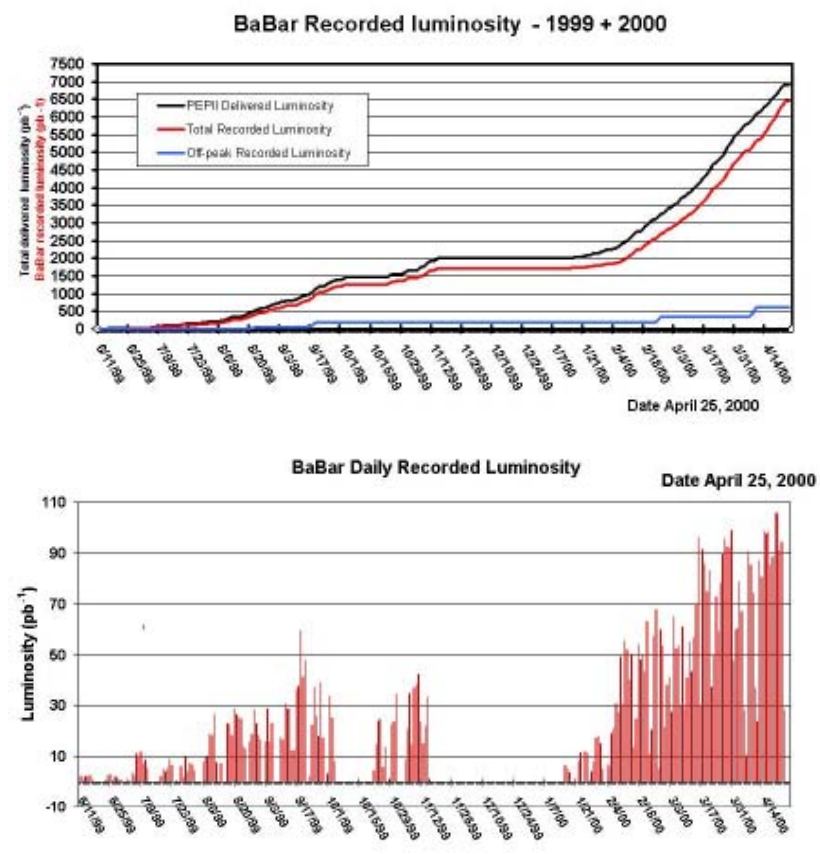

Figure 1. Integrated and daily luminosity history of PEP-II and the BaBar experiment.

the quartz bars for the DIRC particle ID system. The longer inactive period starting in November ' 99 was due to a PEP-II vacuum leak. BaBar has logged $6.5 \mathrm{fb}^{-1}$ of data and now routinely records $100 \mathrm{pb}^{-1}$ in one day.

\section{Performance of the BaBar detector}

The BaBar detector has a fairly standard collider-detector design ${ }^{1}$. At the center of the detector lies the silicon vertex tracker (SVT) which is a 5-layer, double-sided Si device designed to have a $\delta z$ resolution better than $120 \mu \mathrm{m}$. The SVT is a stand-alone tracker, capable of reconstructing tracks down to a $p_{T}$ of $60 \mathrm{MeV}$. The radial region from $23.6 \mathrm{~cm}$ to $81 \mathrm{~cm}$ is occupied by the drift chamber (DCH), which has 10 super layers with 4 cells per super layer. The super layers alternate between axial, U stereo, and $\mathrm{V}$ stereo. Outside the DCH lies the detector of internally reflected Cerenkov radiation (DIRC), which is a powerful particle identification tool unique to the BaBar experi- 
ment. Outside the DIRC lies the CsI(Tl) electromagnetic calorimeter (EMC). The superconducting solenoid flux return is instrumented with resistive plate chambers (RPCs) for muon and neutral hadron identification. BaBar has a two-level trigger, which can operate at output rates of $2 \mathrm{KHz}$ and $100 \mathrm{~Hz}$ for the first and second levels respectively. In the following subsections, we will describe the initial performance of the detector subsystems in more detail.

\subsection{The silicon vertex tracker}

The measured performance of the SVT is consistent with the design specifications. The electronic noise ranges from 800 to 1600 electrons which is well below the signal from a minimum-ionizing particle (about 22000 electrons at normal incidence). Although the PEP-II backgrounds are a bit larger than expected, we see no signs of serious radiation damage. For example, we see less than 10 dead channels due to radiation-induced p-stop shorts out of a total of $152 \mathrm{~K}$ channels. Figure 2 shows the layer 1 hit resolution as measured in data compared to the Monte Carlo simulation. For tracks at normal incidence with the silicon, the hit resolution is about $12 \mu \mathrm{m}$ in both the data and Monte Carlo.

The SVT is mounted on the PEP-II B1 dipole permanent magnets and can move with respect to the DCH by as much as $100 \mu \mathrm{m}$ in a diurnal pattern. In order to maintain our desired resolution, we globally align the SVT with respect to the $\mathrm{DCH}$ after every run as a rolling calibration.

\subsection{The drift chamber}

The drift chamber momentum resolution has been measured with di-muon events to be $d p_{t} / p_{t}=2.9 \% \times p_{t}$ which is consistent with the design specification. Figure 2 shows the DCH hit resolution as a function of position within the cell. The hit resolution ranges from 100 to $200 \mu \mathrm{m}$ with an average value of $125 \mu \mathrm{m}$, which exceeds the design specification of $140 \mu \mathrm{m}$. The DCH $d E / d x$ resolution was measured to be $7.5 \%$ with Bhabha events. We hope to achieve the design specification of $7 \%$ with further corrections.

\subsection{Integrated tracking performance}

A good measure of the integrated tracking performance comes from mass resolutions. Figure 3 shows the mass resolution for $D^{0} \rightarrow K^{-} \pi^{+}$to be $8.8 \pm 1.7$ $\mathrm{MeV} / c^{2}$. The resolution for the narrow core of the $D^{*+}-D^{0}$ mass difference is $243 \pm 7 \mathrm{KeV} / c^{2}$. Figure 4 shows the impact parameter resolution, in the $x y$ and $r z$ planes, as a function of transverse momentum as measured with 

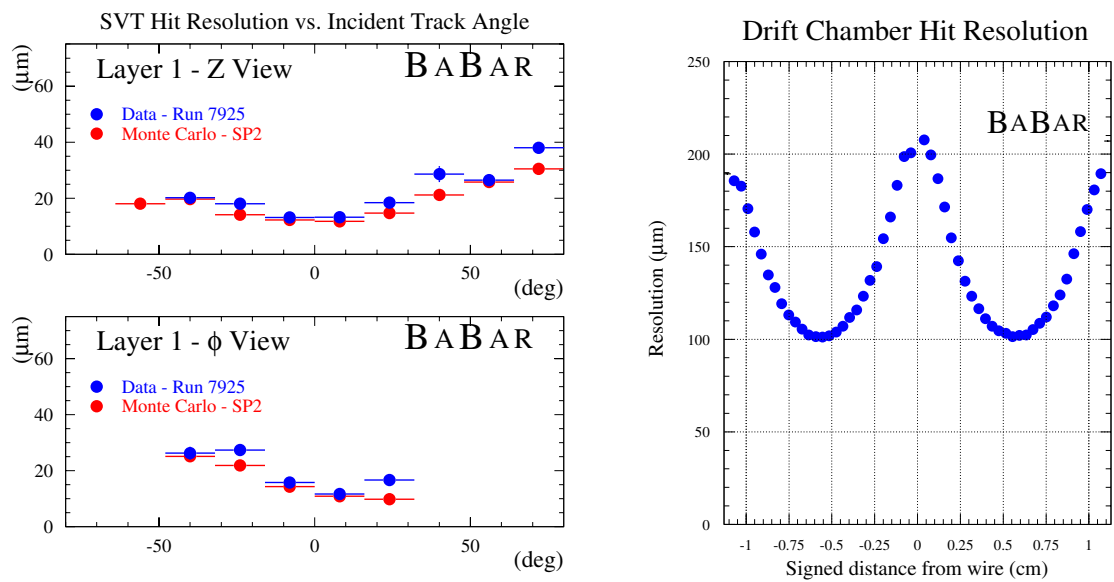

Figure 2. Layer $1 \mathrm{SVT}$ hit resolution as a function of angle of incidence into the silicon and $\mathrm{DCH}$ hit resolution as a function of position within the cell.

multi-hadron events. The asymptotic value at high momentum, for both the $x y$ and $r z$ planes, is about $40 \mu \mathrm{m}$, which is consistent with an independent measurement with di-muon events at high momentum.

\subsection{The detector of internally reflected Čerenkov radiation}

The detector of internally reflected Čerenkov radiation (DIRC) is a powerful particle identification tool that is unique to the BaBar experiment. Cerenkov photons generated in an array of quartz bars that surround the outside of the drift chamber propagate through internal reflections to the end of the detector where they expand in a large water tank and are detected with an array of phototubes. The Cerenkov angle is measured and translates into the particle velocity, which can be used to infer the particle mass with the momentum measurement from the tracking system.

The quartz bars for the DIRC were technically very difficult to produce in large quantities. A typical photon travels $6-10 \mathrm{~m}$ within the quartz with about 300 reflections, so the bars need to have an extremely good polish (surface RMS of 5 - $10 \AA$ ) with sharp edges. Due to these technical challenges, a significant fraction of the bars were delivered 18 months late with a serious cost overrun. 

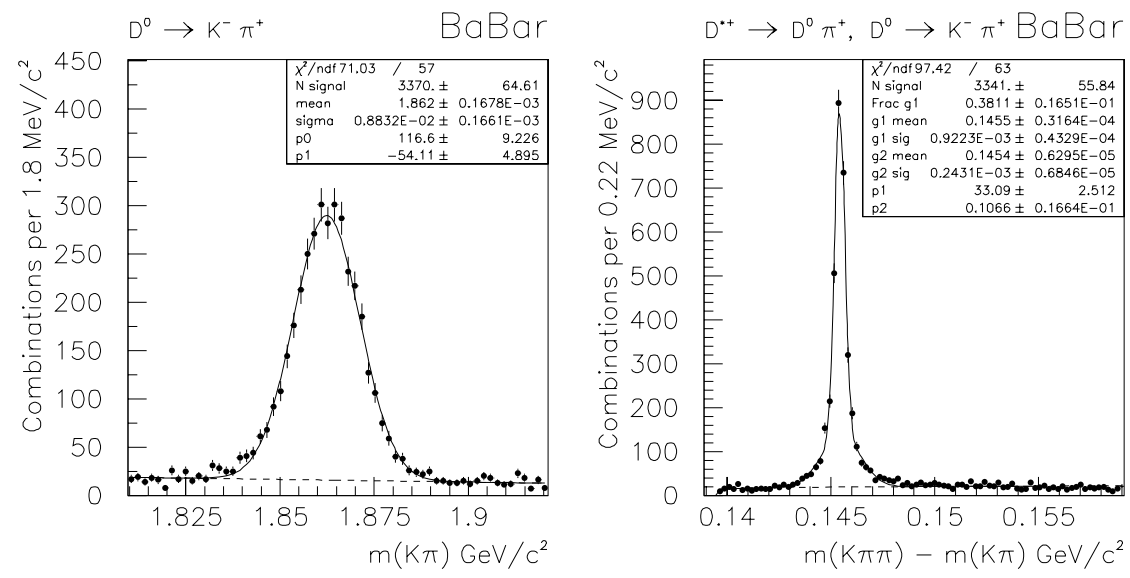

Figure 3. $D^{0}$ and $D^{*+}-D^{0}$ mass resolution.
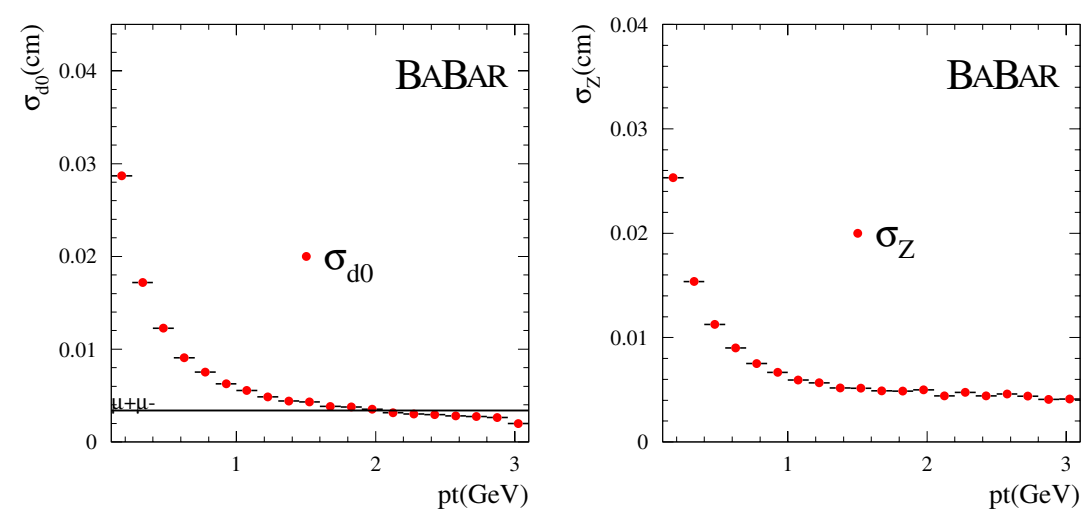

Figure 4. Impact parameter resolution as a function of transverse momentum.

We have measured the Čerenkov angle resolution to be 3.0 mrad with Bhabha events. To give the reader an idea of the necessary resolution, the $\pi-K$ Čerenkov angle difference at $4.0 \mathrm{GeV} / c$ is $6.4 \mathrm{mrad}$. We would like 

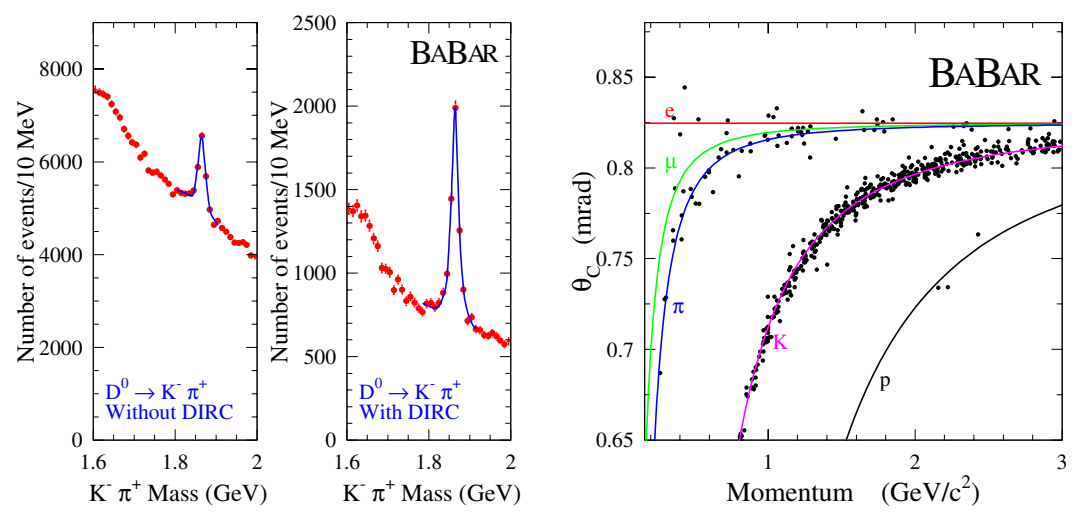

Figure 5. The enhancement in the $D^{0} \rightarrow K^{-} \pi^{+}$signal due to DIRC $K$ identification and the measured Cerenkov angle as a function of momentum for kaons kinematically identified with $D^{0} \rightarrow K^{-} \pi^{+}$.

to eventually achieve a resolution of $2 \mathrm{mrad}$, since good $\pi-K$ separation is required at high momentum for the $C P$ Eigenstate $B^{0} \rightarrow \pi^{+} \pi^{-}$. Figure 5 shows the enhancement in the $D^{0} \rightarrow K^{-} \pi^{+}$signal when the kaon Cerenkov angle is required to be within $6 \mathrm{mrad}(2 \sigma)$ of the expected value for a kaon. The efficiency is about $70-80 \%$ with a rejection factor of about 5 . Figure 5 also shows the Čerenkov angle as a function of momentum for kaons that have been kinematically tagged with the decay chain $D^{*+} \rightarrow D^{0} \pi^{+}, D^{0} \rightarrow K^{-} \pi^{+}$. The combinatoric background from the kinematic selection was about $15 \%$.

\subsection{The electromagnetic calorimeter}

We have measured the resolution on the ratio of the observed energy to the expected energy with Bhabha electrons to be about $2 \%$, which is consistent with the Monte Carlo simulation. Figure 6 shows the reconstructed $\pi^{0} \rightarrow \gamma \gamma$ mass distribution from data, where the minimum $\gamma$ energy is $30 \mathrm{MeV}$ and the minimum $\pi^{0}$ energy is $300 \mathrm{MeV}$. The mass is consistent with the world average and the resolution is $7.4 \mathrm{MeV} / c^{2}$.

\subsection{The instrumented flux return}

We initially had problems in the instrumented flux return (IFR) with high currents in the RPCs due to the iron warming up, especially in the sum- 


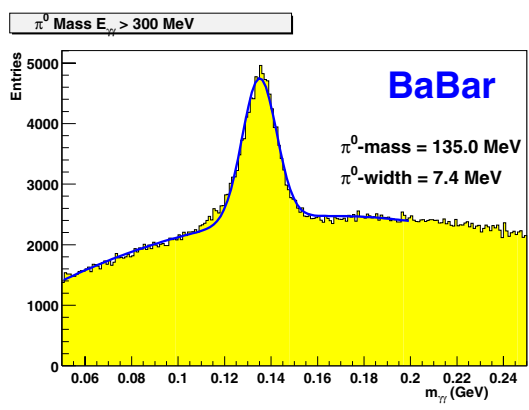

Figure 6. Reconstructed $\pi^{0} \rightarrow \gamma \gamma$ with a minimum $\gamma$ energy of $30 \mathrm{MeV}$ and a minimum $\pi^{0}$ energy of $300 \mathrm{MeV}$.

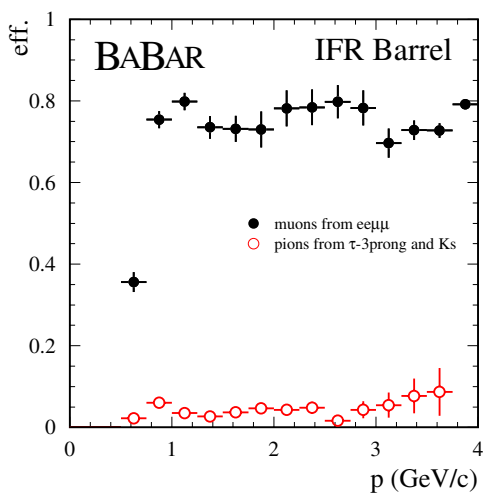

Figure 7. The instrumented flux return muon identification efficiency and pion misidentification fraction as a function of momentum.

mer. Many RPCs were temporarily disconnected before water cooling was installed on the flux return steel. Figure 7 shows the measured IFR muon identification efficiency and the pion misidentification rate as a function of momentum. Above $1 \mathrm{GeV} / c$, the muon ID efficiency is about $75 \%$. The pion misidentification fraction is about $5 \%$. 


\section{Demonstration of physics capability}

The most theoretically clean and experimentally straight-forward $C P$ asymmetry measurement comes from the decay $B^{0} \rightarrow J / \psi K_{s}^{0}$, which gives $\sin (2 \beta)$, where $\beta$ is one of the angles of the Unitarity Triangle. Other experiments have already investigated $C P$ violation with this mode ${ }^{3}$. BaBar plans to make a preliminary measurement of $\sin (2 \beta)$ this summer using an integrated luminosity of about $10 \mathrm{fb}^{-1}$.

Figure 8 shows the inclusive $J / \psi$ signal in the $\mu^{+} \mu^{-}$and $e^{+} e^{-}$channels for an integrated luminosity of about $1.9 \mathrm{fb}^{-1}$. Figure 9 shows the beamconstrained mass distributions for $B^{+} \rightarrow J / \psi K^{+}$and $B^{0} \rightarrow J / \psi K_{s}^{0}, K_{s}^{0} \rightarrow$ $\pi^{+} \pi^{-}$for an integrated luminosity of about $1.9 \mathrm{fb}^{-1}$. The yields are $109 \pm$ 11 and $28 \pm 5$ for the $J / \psi K^{+}$and $J / \psi K_{s}^{0}$ modes respectively, which are consistent with expectations.

A first step towards measuring a proper time dependent $C P$ asymmetry is to measure the $B^{0}$ mixing frequency $\Delta m_{d}$ through the proper time dependence of the flavor oscillations. The charge of the lepton from semileptonic $B$ decay has the same sign as the $b$ quark charge, so $\delta m_{d}$ can be measured by following the number of same-sign and opposite-sign di-lepton events as a function of the longitudinal separation between the $B$ decay vertices $(\delta z)$. Figure 10 shows the dilepton asymmetry as a function of $\delta z$ for selected events from $3 \mathrm{fb}^{-1}$ along with the result of a fit for $\delta m_{d}$. The fitted value of $\delta m_{d}$ is compatible with the world average and the statistical error is about $4 \%$, which is the precision of the ' 98 world average ${ }^{2}$.

BaBar expects to have recorded an integrated on-resonance luminosity of at least $10 \mathrm{fb}^{-1}$ with the full detector by the end of August, 2000. With such a data set, the expected statistical precision of a measurement of $\sin (2 \beta)$ with $B^{0} \rightarrow J / \psi K_{s}^{0}$ is 0.25 .

\section{References}

1. BaBar Technical Design Report, SLAC-R-457 (1995).

2. C. Caso et al. (PDG) Eur. Phys. J. C3 (1998) 1.

3. T. Affolder et al. (CDF) Phys. Rev. D 61, 072005 (2000). K. Ackerstaff et al. (OPAL) Eur. Phys. J. C5 (1998) 379-388.

Also see contributions by G. Apollinari and Andrew Halley in these proceedings. 
Presented at Lake Louise Winter Institute: From Particles To The Universe, 2/20/2000-2/26/2000, Alberta, Canada
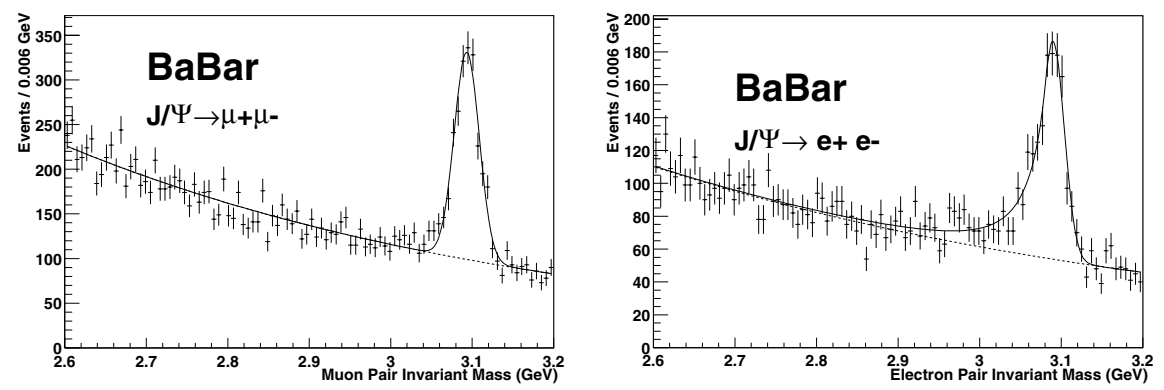

Figure 8. Invariant dilepton mass for the inclusive $J / \psi$ signals in the $\mu^{+} \mu^{-}$and $e^{+} e^{-}$ channels.
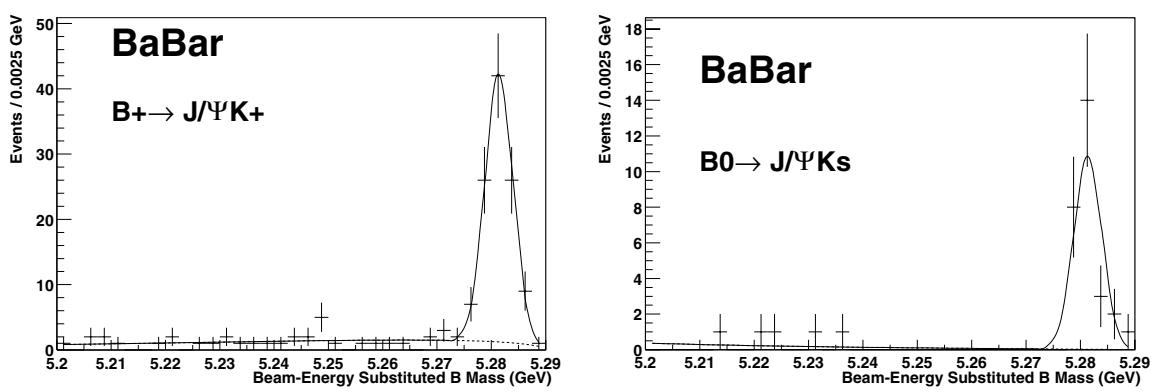

Figure 9. Invariant mass distributions for $B^{+} \rightarrow J / \psi K^{+}$and $B^{0} \rightarrow J / \psi K_{s}^{0}, K_{s}^{0} \rightarrow \pi^{+} \pi^{-}$. 
Presented at Lake Louise Winter Institute: From Particles To The Universe, 2/20/2000-2/26/2000, Alberta, Canada

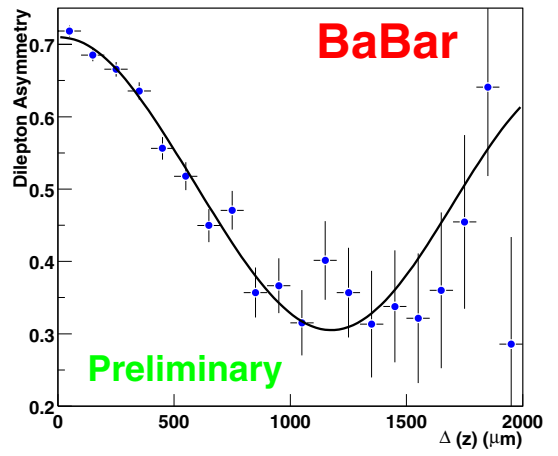

Figure 10. The di-lepton asymmetry as a function of $\delta z$ for selected events. The curve represents the result of a fit for the mixing parameter $\delta m_{d}$. 